\title{
NON-UNIFORM LATTICES ON UNIFORM TREES
}

\section{Lisa Carbone}

\section{Introduction}

Let $G$ be a locally compact group, and $\mu$ a left invariant Haar measure on $G$. A discrete subgroup $\Gamma$ of $G$ is called a $G$ - lattice if $\mu(\Gamma \backslash G)$ is finite, and a uniform (or cocompact) G-lattice if $\Gamma \backslash G$ is compact, non-uniform otherwise.

(1) In the early 1980's, Hyman Bass and Alex Lubotzky proposed to study lattices in the automorphism group of a locally finite tree $X$, a group that is naturally locally compact, in analogy with lattices in non-compact simple real Lie groups. While $G=\operatorname{Aut}(X)$ is not simple, Tits has shown ([Ti]) that when $G$ acts minimally on $X$, fixing no end of $X$, then $G$ has a large simple normal subgroup, $G^{+}$, generated by all edge stabilizers. In fact, when $X$ is homogeneous, $G^{+}$is of index two, so $G$ is 'almost simple'.

(2) The program of Bass and Lubotzky was motivated by the intermediate case of a simple algebraic K-group $H$, of $K$-rank 1 , over a non-archimedan local field $K$, with finite residue field $\mathbb{F}_{q}$. The group $H \leq A u t(X)$ acts on its Bruhat-Tits tree $X$; for example, if $H=P S L_{2}(K)$ then $X$ is the homogeneous tree $X_{q+1}$.

(3) With respect to this program of study, many of the natural questions have been treated by Bass, Lubotzky, R. Kulkarni and others. Some examples are: the existence of uniform tree lattices $([\mathrm{BK}])$, and of non-uniform tree lattices ([BCR], $[\mathrm{C} 2]$, $[\mathrm{CR} 1])$, the structure of uniform and non-uniform tree lattices $([\mathrm{BK}],[\mathrm{BL}])$, covolumes $([\mathrm{BK}],[\mathrm{BL}]$, $[\mathrm{IL}],[\mathrm{R}])$, commensurability groups of uniform tree lattices ([BK], $[\mathrm{YL}]$ ), super-rigidity ([LMZ], $[\mathrm{BM}])$, the congruence subgroup problem for uniform lattices on regular trees ([Mo]), and the existence of towers of lattices ([CR2], [CR3], [R]).

(4) R. Kulkarni, in $[\mathrm{K}]$, has also indicated an analogy of the study of trees and their lattices with the study of discontinuous groups and Riemann surfaces, as well as direct connections with automorphisms of graphs, free groups and surfaces, and with the structure of finite groups.

Received by the editor January 27, 1998 
(5) For the study of tree lattices in analogy with lattices in Lie groups, many interesting questions as yet remain open, such as the arithmeticity and commensurability of nonuniform tree lattices, the congruence subgroup problem on non-homogeneous trees, and for non-uniform lattices, as well as direct connections between tree lattices and lattices in rank 1 Lie groups over non-archimedean local fields. We refer the reader to [L1] for a survey of the comparisons between tree lattices and lattices in Lie groups.

(6) The present work contains a proof of a conjecture about the existence of non-uniform lattices on 'uniform trees'. Bass and Lubotzky conjectured, in analogy with Borel's theorems in the classical case about the co-existence of uniform and non-uniform lattices in connected non-compact semisimple Lie groups ([Bo1], [Bo2]), that when uniform lattices are present in $G=\operatorname{Aut}(X)$, under some natural assumptions, there should also be nonuniform lattices.

(7) In the case that $X$ is the Bruhat-Tits tree of a rank 1 simple Lie group $\mathrm{H}$ over a non-archimedean local field $K$ of characteristic $p>0$, the existence of (both arithmetic and non-arithmetic) uniform and non-uniform lattices in $H$ was established by Alex Lubotzky in [L2]. If $K$ has characteristic zero, it is well known ([Ta]) that $H$ cannot contain non-uniform lattices, while the existence of arithmetic uniform lattices in $H$ was proven by Borel and Harder $([\mathrm{BH}])$. In [L2], Lubotzky showed that $H$ contains non-arithmetic uniform lattices as well.

This work concerns the existence of non-uniform lattices in the automorphism group of a general locally finite tree. In order to discuss the precise statements and strategy, we introduce some terminology.

(8) Let $X$ be a locally finite tree, and $G=A u t(X)$ its group of automorphisms. The stabilizers $G_{S}$ of finite sets, $S \subset V X$, of vertices form a fundamental system of neighborhoods of the identity. In particular, the stabilizer $G_{x}$ of a vertex $x \in V X$ is compact and open, in fact, profinite and hence totally disconnected. Thus $G=\operatorname{Aut}(X)$ is locally compact and totally disconnected.

(9) A subgroup $\Gamma \leq G$ is discrete if and only if all vertex stabilizers $\Gamma_{x}$ are finite. In 
this case, we define a volume:

$$
\operatorname{Vol}(\Gamma \backslash \backslash X) \quad:=\sum_{x \in V(\Gamma \backslash X)} 1 /\left|\Gamma_{x}\right|,
$$

and call $\Gamma$ an $X$-lattice if $\operatorname{Vol}(\Gamma \backslash \backslash X)$ is finite, and a uniform $X$-lattice if the quotient graph $\Gamma \backslash X$ is finite. Thus a non-uniform $X$-lattice is a discrete subgroup $\Gamma$ of $G$ with infinite quotient graph $\Gamma \backslash X$ but finite covolume $\operatorname{Vol}(\Gamma \backslash \backslash X)$.

(10) Locally finite trees which admit uniform lattices are exactly the universal covers of finite connected graphs; these are called uniform trees ([BK]). When $X$ is uniform, a uniform (respectively non-uniform) $X$-lattice is a uniform (respectively non-uniform) $G$-lattice, and conversely ([BL]). We wish to understand when a uniform tree $X$ admits a non-uniform $X$ - (or $G$-) lattice.

(11) For $X$ to admit a uniform $X$-lattice, it is necessary and sufficient that $G=\operatorname{Aut}(X)$ be unimodular, and that $G \backslash X$ be finite ([BK]). Bass and Tits have shown ([BT]) that there are many uniform trees whose automorphism group $G$ is discrete and hence is itself a uniform lattice, so it cannot contain a non-uniform lattice. Moreover, there are examples ([BL], and $c f$. section 3 ) where $G$ is not discrete, but its action on $X$ is not minimal (that is; there is a proper $G$-invariant subtree), and all $X$-lattices are uniform. We have the following, conjectured in an earlier version of [BL]:

(12) Conjecture. Let $X$ be a locally finite tree, and $G=\operatorname{Aut}(X)$ its group of automorphisms. Suppose that $G$ is unimodular and $G \backslash X$ is finite (thus, $X$ admits a uniform $X$-lattice). If $G$ is not discrete and acts minimally on $X$, then there is a non-uniform $X$-lattice $\Gamma$ in $G$.

(13) We present here a proof of conjecture 0.12. Our proof is 'constructive'; under the above conditions, we exhibit a non-uniform lattice $\Gamma$ in $G$. Our strategy is to use a type of 'inverse Bass-Serre theory'; that is, we construct the quotient graph of groups $\Gamma \backslash \backslash X$ for the action of $\Gamma$ on $X$.

(14) If $\mathbb{A}=(A, \mathcal{A})$ is a graph of groups with underlying graph $A$, vertex groups $\mathcal{A}_{a}$, edge groups $\mathcal{A}_{e}$ and monomorphisms $\alpha_{e}: \mathcal{A}_{e} \hookrightarrow \mathcal{A}_{\partial_{0}(e)}$, we put $i(e)=\left[\mathcal{A}_{\partial_{0}(e)}: \alpha_{e} \mathcal{A}_{e}\right]$ for each (oriented) edge $e \in E A$. The graph $(A, i)$ consisting of $A$ and the indices $i(e)$ 
labelling each $e \in E A$ is called the edge-indexed graph $(A, i)=I(\mathbb{A})$ for the graph of groups $\mathbb{A}$. An edge $e$ is called ramified if $i(e)>1$, and unramified otherwise.

(15) We form the graph of groups $G \backslash \backslash X$ for the action of $G=A u t(X)$ on $X$, and the corresponding edge-indexed quotient graph $(A, i)=I(G \backslash \backslash X)$.

(16) We restate our conditions on $G$ and $X$ in terms of the edge-indexed quotient graph $(A, i)=I(G \backslash \backslash X)$.

(17) The existence of a non-uniform $X$-lattice $\Gamma \leq G$ then corresponds to the existence of an infinite covering $p:(B, j) \longrightarrow(A, i)$ of edge-indexed graphs (in the sense of 2.4) for some indexed graph $(B, j)$, such that $(B, j)$ has 'finite volume'. The non-uniform $X$-lattice $\Gamma$ will be the fundamental group $\pi_{1}\left(\mathbb{B}, b_{0}\right)$ of a finite 'grouping' $\mathbb{B}$ of $(B, j)$. Such finite groupings do not automatically exist, but $[\mathrm{BK}]$ give necessary and sufficient conditions for their existence. Namely, we require that $(B, j)$ satisfy the combinatorial conditions 'unimodular' and 'bounded denominators' (see 1.4). Once these conditions are satisfied, there is automatically a finite 'cyclic' grouping of $(B, j)$ (see 1.4), however, any finite ('faithful') grouping is allowable (see 1.3); this gives our technique for constructing lattices a great deal of flexibility. Finally, the fact that $\Gamma$ is non-uniform is reflected in the condition that $(B, j)$ is infinite.

(18) We will thus have proven the conjecture if we can exhibit infinite coverings $p$ : $(B, j) \longrightarrow(A, i)$ with these properties for all the possible edge-indexed quotient graphs $(A, i)=I(G \backslash \backslash X)$ that can occur.

(19) The following theorem of Bass and Lubotzky ([BL]) indicates that the geometry of the quotient of a tree by a lattice is essentially arbitrary:

Theorem ([BL]). Let $A$ be any connected locally finite graph. Then there exists a locally finite tree $X$ and an $X$-lattice $\Gamma$ such that $A=\Gamma \backslash X$. $\square$

(20) We say that an indexed graph $(A, i)$ is minimal, if $(A, i)$ has no terminal vertices; that is, $\operatorname{deg}_{(A, i)}(v)>1$ for every $v \in V A$. In case $(A, i)$ is minimal, we say that $(A, i)$ is non-discretely ramified, if there exists $e \in E A$ such that $i(e) \geq 3$ or $i(e)=2$ and $E_{0}\left(\partial_{0}(e)\right)\left(=\left\{f \in E A \mid \partial_{0}(f)=\partial_{0}(e)\right\}\right) \neq\{e\}$; this is the criterion of Bass and Tits that ensures that the automorphism group of the covering tree is not discrete, ( $c f$. section 3 ). 
The (combinatorial) statement of our main theorem is the following:

(21) Theorem. Let $(A, i)$ be any connected edge-indexed graph. Suppose that $(A, i)$ is finite, unimodular, non-discretely ramified and minimal. Then $(A, i)$ has a covering of edge-indexed graphs $p:(B, j) \longrightarrow(A, i)$ such that $(B, j)$ is infinite, (hence p has infinite fibers), unimodular, has finite volume and bounded denominators.

(22) Our proof is essentially as follows: we first prove Theorem 0.21 in the case that $(A, i)$ contains an 'arithmetic bridge' $\beta$; that is, a set of $n \geq 2$ edges whose removal separates $A$ into two connected components, and where the indices on the positively oriented end of the bridge all have a common factor $d>1$ (section 4 ). In this case, there is a covering with the desired properties (see fig 0.22). A detailed description of the construction of such coverings, including examples, is given in [C3] (cf. section 4).

(23) We then prove Theorem 0.21 in the cases that $(A, i)$ has a 'good' ramified separating edge (section 5), or has a ramified loop (section 6). In order to allow for the possibility that the indexed quotient contains multiple edges, we define a 'simplification' of a graph with no loops (section 7), which naturally has the property that for any edge $e$, the indices $i(e)$ and $i(\bar{e})$ are relatively prime. We also describe a method for treating unramified loops (section 8.1). In view of the cases treated, we can then assume that the quotient graph has no loops or multiple edges.

(24) To give a complete proof of conjecture 0.12 , we need an 'existence theorem' for arithmetic bridges. The remainder of our argument roughly states that every indexed graph $(A, i)$ with no loops or multiple edges, satisfying our original hypothesis must contain an arithmetic bridge or a 'good' separating edge.

(25) We prove this theorem in three stages: we embed $(A, i)$ into the unique complete graph with the same vertices, and with new edges indexed so as to preserve unimodularity and relatively prime indices (section 8.2). We prove, using iterated 'suspensions', that the complete indexed graph must contain an arithmetic bridge (section 8.3), and finally, that the 'restriction' of the arithmetic bridge in the complete graph to the original graph yields an arithmetic bridge for the original (section 8.4). This sequence of arguments gives a complete proof of conjecture 0.12 . 
(26) We have obtained sufficient conditions for the existence of non-uniform lattices on a uniform tree $X$. In order to construct a non-uniform $X$-lattice, it is necessary to assume that $G=\operatorname{Aut}(X)$ is unimodular and not discrete. The requirement that $G$ acts minimally on $X$ is sufficient but not necessary. Following ([BL]) we call $X$ rigid if $G$ is discrete, and we call $X$ minimal if $G$ acts minimally on $X$, that is, there is no proper $G$-invariant subtree. If $X$ is uniform then there is always a unique minimal $G$-invariant subtree $X_{0} \subseteq X$ ([BL]). We call $X$ virtually rigid if $X_{0}$ is rigid ([BL]). All lattices on virtually rigid trees must be uniform ([BL]). Conversely, in the case that $X$ is not minimal, it is proven in [C2] that if $X$ is uniform and not virtually rigid then $G$ contains a non-uniform $X$-lattice $\Gamma$.

For $x \in V X$ we have $0<\mu\left(G_{x}\right)<\infty$, where $\mu$ is a (left) Haar measure on $G$. When $G$ is unimodular, $\mu\left(G_{x}\right)$ is constant on $G$-orbits, so we can define ([BL]):

$$
\mu(G \backslash \backslash X) \quad:=\sum_{x \in V(G \backslash X)} \frac{1}{\mu\left(G_{x}\right)} .
$$

In [BCR] we prove the 'Lattice Existence Theorem', namely that $G$ contains an $X$-lattice $\Gamma$ if and only if $G$ is unimodular and $\mu(G \backslash \backslash X)<\infty$. In particular, it is shown in [BCR] that if $G$ is unimodular, $\mu(G \backslash \backslash X)<\infty$, and $G \backslash X$ is infinite, then $G$ contains a (necessarily non-uniform) $X$-lattice $\Gamma$, which is a uniform $G$-lattice. For non-uniform $G$-lattices, in [CR1] we show that if $X$ has more than one end, and if $G$ contains a non-uniform $X$-lattice, then $G$ contains a non-uniform $G$-lattice. In case $X$ is a uniform tree, this result follows from the present work. Conversely, in [CC], we investigate the question as to the existence of non-uniform $G$-lattices in the case that $X$ has a unique end, and $G$ contains a non-uniform $X$-lattice.

(27) We have constructed a non-uniform $X$-lattice $\Gamma$ by taking a finite grouping of our infinite edge-indexed graph $(B, j)$, which is a 'non-uniform covering' of the indexed quotient graph $(A, i)=I(G \backslash \backslash X)$, where $G=A u t(X)$. Our construction is such that the indexed graph $(B, j)$ satisfies $[\mathrm{BK}]$ 's necessary and sufficient conditions for the existence of finite groupings of $(B, j)$, (that $(B, j)$ be 'unimodular' and have 'bounded denominators' (see 1.4)). We have thus ensured that a finite cyclic grouping of $(B, j)$ exists. The 
question as to how many finite faithful groupings an edge-indexed graph admits, up to isomorphism, is a complex and interesting question. In [CR2] and [CR3], it is shown that if $X$ has more than one end, and if $(B, j)$ admits a finite faithful grouping, then $(B, j)$ admits an infinite tower of finite faithful groupings. This also implies that for the corresponding lattices, there is no lower bound on the covolume.

(28) With respect to the question of arithmeticity of tree lattices, we may adopt (see [BL], [L1]) a criterion of Margulis for detecting arithmeticity; that an $X$-lattice $\Gamma$ is arithmetic if and only if its commensurability group $C_{G}(\Gamma)=\left\{g \in G \mid g \Gamma g^{-1} \sim_{\text {comm }} \Gamma\right\}$ is dense in $G=A u t(X)$. Y.S. Liu's Uniform density theorem ([YL]) states that if $\Gamma$ is a uniform $X$-lattice, then $C_{G}(\Gamma)$ is dense in $G$, and so by this criterion, all uniform $X$-lattices are arithmetic. This is not surprising, as all uniform $X$-lattices are commensurable up to conjugation by an element of $G=\operatorname{Aut}(X)$ ([BK]). It is known ([Mo2]) that the commensurability group $C_{G}(\Gamma)$ of $\Gamma=P S L_{2}\left(\mathbb{F}_{q}[t]\right)$, the 'characteristic $p$ modular group', is dense in $G=\operatorname{Aut}\left(X_{q+1}\right)$, where $X_{q+1}$ is the Bruhat-Tits tree of $P S L_{2}\left(\mathbb{F}_{q}\left(\left(t^{-1}\right)\right)\right)$. The group $P S L_{2}\left(\mathbb{F}_{q}[t]\right)$ is well known to be an arithmetic non-uniform lattice, in the classical sense, as a subgroup of its ambient Lie group $P S L_{2}\left(\mathbb{F}_{q}\left(\left(t^{-1}\right)\right)\right)$. We also know of examples ([BL], [Mo2]) of non-uniform $X_{q+1}$-lattices $\Phi$ such that $\Phi<G-H$, where $G=\operatorname{Aut}\left(X_{q+1}\right), H=P S L_{2}\left(\mathbb{F}_{q}\left(\left(t^{-1}\right)\right)\right)$ and $C_{G}(\Gamma)$ is discrete. The structure of the commensurability group of a general non-uniform $X$-lattice is not currently known.

(29) We survey some of the properties of the non-uniform lattices constructed here, and we refer the reader to [BL] for proofs and for further details. Let $X$ be a locally finite tree, and let $\Gamma$ be a non-uniform $X$-lattice. Then $\Gamma$ is not finitely generated; the vertex stabilizers $\Gamma_{x}$, for $x \in X$, are arbitrarily large subgroups of $\Gamma$, so $\Gamma$ cannot be virtually free, or equivalently, $\Gamma$ cannot be virtually torsion free.

(30) It is known $([\mathrm{VH}])$ that automorphism groups of trees, and hence their lattices, do not satisfy Kazhdan's property T.

(31) The Any quotient theorem of ([BL], Thm 4.17) (cf. 0.19) indicates that any locally finite graph can occur as the quotient graph $\Gamma \backslash X$. Moreover, the number of 'cusps' of $\Gamma \backslash X$ may be finite or infinite, and $\Gamma \backslash X$ may have any geometric cusp structure that is 
'combinatorially allowable' ([BL], 4.13). The rank of the free group $\pi_{1}(\Gamma \backslash X)$ may be any cardinal $\leq \aleph_{0}([\mathrm{BL}], 4.11)$

(32) If $X$ is the Bruhat-Tits tree of a rank 1 simple Lie group H over a non-archimedean local field $K$ of characteristic $p>0$, we know by Lubotzky's theorem ([L2]) that $H$ contains uncountably many conjugacy classes of non-uniform lattices. By the nonuniform existence theorem proven here, we know that there are non-uniform lattices inside $G=A u t(X)$ which contains $H$ as a proper and 'relatively small' subgroup. Our construction of non-uniform $X$-lattices is combinatorial in nature, and will in general not place our lattices inside the Lie group $H$. By strengthening the coverings of edge-indexed quotient graphs developed here to covering morphisms of quotient graphs of groups with the desired properties, we can use a technique of Hyman Bass ([B]) to try to construct non-uniform lattices actually contained within $H$. We hope to address this elsewhere. (33) In $[\mathrm{BL}]$, for homogeneous trees, and in $[\mathrm{R}]$, for uniform trees that admit nonuniform $X$-lattices, it is shown that for every real number $v>0$, there is an $X$-lattice $\Gamma$ such that $\operatorname{Vol}(\Gamma \backslash \backslash X)=v$. Since the covolume of an $X$-lattice is constant on its conjugacy class, we may deduce that if $X$ is a uniform tree that admits a non-uniform $X$-lattice, then $X$ admits uncountably many conjugacy classes of non-uniform $X$-lattices.

The author is indebted to her PhD thesis adviser, Hyman Bass, whose careful attention and untiring efforts have played a substantial role in the development of this work. The author has great pleasure in thanking him.

Cambridge, Massachusetts, August 2000

\section{BIBLIOGRAPHY}

[B] Bass H, Covering theory for graphs of groups, Journal of Pure and Applied Algebra 89 (1993).

[BCR] Bass, H, Carbone L, and Rosenberg, G, The Existence Theorem for tree lattices, Appendix B, 'Tree Lattices' by Hyman Bass and Alex Lubotzky (2000), Birkhauser, Boston.

[Bo1] Borel, A, Introduction aux groupes arithmetiques, Herman, Paris (1990).

[Bo2] Borel, A, Compact Clifford-Klein forms of symmetric spaces, Topology 2 (1963).

[BH] Borel, A, Harder, G, Existence of discrete cocompact subgroups of reductive groups over local fields, J. Reine Angew. Math 298 (1978).

[BK] Bass H and Kulkarni R, Uniform tree lattices, Journal of the Amer Math Society 3 (4) (1990).

[BL] Bass H and Lubotzky A, Tree lattices (2000), Birkhauser, Boston. 
[BM] Burger, M and Mozes, S, CAT(-1) Spaces, divergence groups and their commensurators, Preprint.

[BT] Bass H and Tits J, A Discreteness Criterion for certain tree automorphism groups, Appendix A, 'Tree Lattices' by Hyman Bass and Alex Lubotzky (2000), Birkhauser, Boston.

[C1] Carbone L, Non-uniform lattices on uniform trees, PhD. Thesis, Columbia University (1997).

[C2] Carbone, L, Non-minimal tree actions and the existence of non-uniform tree lattices, (in preparation) (2000).

[C3] Carbone L, Constructing tree lattices, Algebras and Combinatorics. An International Congress, ICAC '97, Hong Kong (ed K.P. Shum and E. Taft) (pp 63-97) (1999), Springer.

[CC] Carbone, L and Clark, D, Lattices on parabolic trees, Preprint (2000).

[CR1] Carbone, L and Rosenberg, G, Lattices on non-uniform trees, Preprint (2000).

[CR2] Carbone, L and Rosenberg, G, Infinite towers of tree lattices, Preprint (2000).

[CR3] Carbone, L and Rosenberg, G, Infinite towers of non-uniform tree lattices, (in preparation) (2000).

[K] Kulkarni R, Lattices on trees, automorphisms of graphs, free groups and surfaces, Preprint (1993).

[IL] Levich, Inga, PhD thesis, Hebrew University of Jerusalem (1996).

[L1] Lubotzky A, Tree lattices and lattices in Lie groups, in Combinatorial and Geometric Group Theory, ed A. Duncan, N. Gilbert and J. Howie, LMS Lecture Note Series 204 (pp 217-232) (1995), Cambridge University Press.

[L2] Lubotzky A, Lattices in rank one Lie groups over local fields, Geometric and Functional Analysis 4 (pp 405-431) (1991).

[L3] Lubotzky A, Lattices of minimal covolume in $S L_{2}$, Jour of the AMS 3 (pp 961-975) (1990).

[LMZ] Lubotzky, A, Mozes, S and Zimmer, R, Superrigidity for the commensurability group of tree lattices, Comment. Math. Helvetici 69 (pp 523-548) (1994).

[Ma] Margulis, G, Discrete subgroups of semi-simple Lie groups, Springer-Verlag (1991).

[Mo] Mozes, S, The congruence subgroup problem for uniform tree lattices, Preprint.

[Mo2] Mozes, S, Private communication (1997).

[R] Rosenberg, G, Infinite towers of uniform tree lattices, PhD. Thesis, Columbia University (2000).

[S] Serre, J.P, Trees (Translated from the French by John Stilwell) (1980), Springer-Verlag, Berlin Heidelberg.

[Ta] Tamagawa, T, On discrete subgroups of p-adic algebraic groups, Arithmetical Algebraic Geometry (ed O. F. G. Schilling) (1965), Harper and Row.

[Ti] Tits, J, Sur le groupe des automorphisms d'un arbe, Essays on topology and related topics: Memories dedies a George de Rham (1970), Springer.

[VH] Valette, A and de la Harpe, P, La propriete $(T)$ de Kazhdan pour les groupes localement compacts (avec un appendice de Marc Burger), Asterisque 175 (1989).

[YL] Liu, YS, Density of the commensurability group of uniform tree lattices, Journal of Algebra 165 (1994).

Department of Mathematics, Harvard University, Science Center 325, 1 Oxford St, Cambridge MA 02138

e-mail lisa@ math.harvard.edu 